\title{
ON SOME DEFORMATIONS OF RIEMANN SURFACES. I
}

\author{
RYSZARD L. RUBINSZTEIN
}

\begin{abstract}
We define a family of infinitesimal deformations of compact Riemann surfaces of genus $g \geq 2$ which generalizes the Fenchel-Nielsen deformations. Those new deformations are associated to smooth vector fields on the circle. We compute a representation of the deformations in terms of Poincare series and determine the corresponding Eichler cohomology classes.
\end{abstract}

Let $R$ be a compact Riemann surface (a complex manifold of complex dimension 1) of genus $g \geq 2$. Let $C$ be a simple closed geodesic on $R$ (with respect to the hyperbolic metric).The Fenchel-Nielsen deformation of $R$ is obtained by cutting $R$ along the geodesic $C$, rotating one side of the cut by some angle $\alpha$ and then regluing both sides of the cut in their new position. When the angle $\alpha$ is allowed to converge to 0 , one obtains the infinitesimal Fenchel-Nielsen deformation. This deformation has been extensively studied, see e.g. [5], [6].

In this paper we introduce a new family of infinitesimal deformations of $R$ generalizing that of Fenchel-Nielsen.

Let $\mathfrak{X}$ be a smooth vector field on the circle $S^{1}$. Let $C_{0}$ and $C_{1}$ be a pair of geodesics on $R$ which intersect in one point. Given those data, we construct an infinitesimal deformation $\varphi_{\left(C_{0}, C_{1}\right)}(\mathfrak{X})$ of $R$. The geometric meaning of the deformation is as follows: the vector field $\mathfrak{X}$ on $S^{1}$ generates a 1-parameter group of diffeomorphisms $f_{t}$ of $S^{1}$ (the flow of $\mathfrak{X}$ ). Identify the geodesic $C_{0}$ with $S^{1}$ (the intersection point of $C_{0}$ with $C_{1}$ is identified with $1 \in S^{1}$ ). Cut the surface $R$ along $C_{0}$, change the position of one side of the cut by the diffeomorphism $f_{t}$ and reglue both sides of the cut in their new position. When $t$ converges to 0 one obtains an infinitesimal deformation $\varphi_{\left(C_{0}, C_{1}\right)}(\mathfrak{X})$ of the surface $R$.

In the special case when the vector field $\mathfrak{X}$ on $S^{1}$ is the constant one, $\mathfrak{X}=\frac{\widehat{d}}{d x}$ (see Sec.1), the 1-parameter group of diffeomorphisms $f_{t}$ is the group 
of rotations of the circle and our construction gives the infinitesimal Fenchel-Nielsen deformation based on the geodesic $C_{0}$.

The contents of the paper are as follows: in Section 2 we construct the infinitesimal deformation $\varphi_{\left(C_{0}, C_{1}\right)}(\mathfrak{X})$ and compute the Beltrami differential $\nu=\nu(\mathfrak{X})$ which represents it. In Section 3 we describe the deformation in terms of quadratic differentials in the lower half-plane $\mathrm{H}^{*}$. This is done for the case when $\mathfrak{X}$ has a finite Fourier expansion. The quadratic differential is given by a Poincaré series. The main result is Theorem 3.7. In Section 4 we give a description of the Eichler cohomology class which corresponds to our deformation (again for $\mathfrak{X}$ with a finite Fourier expansion). Results of Sections 3 and 4 generalize some of the results of S. Wolpert, [5], for the Fenchel-Nielsen deformation. Finally, in Section 5 we point out that the infinitesimal deformation $\varphi_{\left(C_{0}, C_{1}\right)}(\mathfrak{X})$ defines a vector field $\Phi_{\left(C_{0}, C_{1}\right)}(\mathfrak{X})$ on the Teichmüller space $T(R)$ of $R$.

We construct our deformations in the context of quasiconformal mappings. For the background material on quasiconformal mappings and Teichmüller spaces we refer to [2].

\section{Vector fields on $S^{1}$}

Let $S^{1}$ be a circle. We look upon $S^{1}$ as the unit circle in the complex plane,

$$
S^{1}=\{z \in \mathrm{C}|| z \mid=1\} .
$$

Let $\mathfrak{X}$ be a smooth tangent vector field on $S^{1}$. $\mathfrak{X}$ determines a 1-parameter group of diffeomorphisms of $S^{1}$,

$$
f_{t}: S^{1} \longrightarrow S^{1}, \quad t \in \mathrm{R},
$$

with $f_{t} \circ f_{s}=f_{t+s}, \quad f_{0}=\mathrm{id}_{S^{1}}$ and such that $\left.\frac{d}{d t} f_{t}(z)\right|_{t=0}=\mathfrak{X}(z)$ for $z \in S^{1}$.

Let $p: \mathrm{R} \longrightarrow S^{1}, p(x)=e^{2 \pi i x}$. The map $p$ is a universal covering of $S^{1}$. By the Covering Homotopy Property of $p$ there exists a unique lifting of $\left\{f_{t}\right\}_{t \in \mathrm{R}}$ to a 1-parameter family of smooth maps

$$
\tilde{f}_{t}: \mathrm{R} \longrightarrow \mathrm{R}
$$

satisfying $p \circ \tilde{f}_{t}=f_{t} \circ p$ for $t \in \mathrm{R}$ and $\tilde{f}_{0}=\mathrm{id}_{\mathrm{R}}$.

By a standard unique path lifting argument it follows then that $\tilde{f}_{t} \circ \tilde{f}_{s}=\tilde{f}_{t+s}$ for all $t, s \in \mathrm{R}$, hence the lifting $\tilde{f}_{t}: \mathrm{R} \longrightarrow \mathrm{R}$ is a 1-parameter group of diffeomorphisms of R.

Since $p: \mathbf{R} \longrightarrow S^{1}$ is a local diffeomorphism, there exists a unique tangent 
vector field $\tilde{\mathfrak{X}}$ on $\mathbf{R}$ such that $d p_{x}(\tilde{\mathfrak{X}}(x))=\mathfrak{X}(p(x))$ for all $x \in \mathbf{R}$. It is clear that

$$
\left.\frac{d}{d t}\left(\tilde{f}_{t}(x)\right)\right|_{t=0}=\tilde{\mathfrak{X}}(x), \quad x \in \mathbf{R} .
$$

Hence $\left\{\tilde{f}_{t}\right\}_{t \in \mathrm{R}}$ is the 1-parameter group of diffeomorphisms of $\mathrm{R}$ generated by the vector field $\tilde{\mathfrak{X}}$.

For every $t \in \mathrm{R}$ the map $f_{t}: S^{1} \longrightarrow S^{1}$ is homotopic to identity, hence $\operatorname{deg}\left(f_{t}\right)=1$. It follows that

$$
\tilde{f}_{t}(x+1)=\tilde{f}_{t}(x)+1
$$

for all $t, x \in \mathrm{R}$.

Moreover, for every $t \geq 0$ there are real constants $\alpha_{t}^{\prime}, \alpha_{t}^{\prime \prime}>0$ such that

$$
\alpha_{t}^{\prime} \leq\left|\frac{d}{d x} \tilde{f}_{s}(x)\right| \leq \alpha_{t}^{\prime \prime}
$$

for all $x \in \mathrm{R}$ and all $s \in \mathrm{R}$ with $|s| \leq t$. There is also a real constant $M>0$ such that

$$
\left|\frac{d}{d t} \tilde{f}_{t}(x)\right| \leq M
$$

for all $x, t \in \mathrm{R}$.

$\frac{d}{d x}$ is a nowhere vanishing vector field on R. Via the map $p$ it descends to a vector field on $S^{1}$ which we denote by $\frac{d}{d x}$. Hence, in our notation

$$
\frac{\widetilde{d}}{d x}=\frac{d}{d x} .
$$

Every smooth vector field $\mathfrak{X}$ on $S^{1}$ can now be written as $\mathfrak{X}=h \frac{\widehat{d}}{d x}$, with $h: S^{1} \longrightarrow \mathrm{R} \quad$ being a smooth function. Then $\tilde{\mathfrak{X}}=\tilde{h} \frac{d}{d x} \quad$ with $\tilde{h}: \mathbf{R} \longrightarrow \mathbf{R}, \tilde{h}=h \circ p$. Note that $\tilde{h}(x+1)=\tilde{h}(x)$.

Remark 1.4. Let Diff $+\left(S^{1}\right)$ be the group of orientation preserving diffeomorphisms of $S^{1}$. Considered as a topological space (with a suitable topology, see [4]) $\operatorname{Diff}_{+}\left(S^{1}\right)$ is not simply-connected. Let $\operatorname{Diff}_{1}^{\text {per }}(\mathrm{R})$ be the space of all diffeomorphisms $g: \mathrm{R} \longrightarrow \mathrm{R}$ satisfying

$$
g(x+1)=g(x)+1 \quad \text { for all } x \in \mathbf{R} .
$$


$\operatorname{Diff}_{1}^{\text {per }}(R)$ is a group with respect to composition. There is a continuous map

$$
\pi: \operatorname{Diff}_{1}^{\text {per }}(\mathrm{R}) \longrightarrow \operatorname{Diff}_{+}\left(S^{1}\right)
$$

given by $\pi(g)(z)=p(g(x))$ for any $z \in S^{1}$ and $x \in p^{-1}(z)$. The map $\pi$ is a group homomorphism and a covering map.

Moreover, as a topological space $\operatorname{Diff}_{1}^{\text {per }}(R)$ is contractible. Indeed, a contraction of $\operatorname{Diff}_{1}^{\text {per }}(R)$ to a point is given by

$$
H: \operatorname{Diff}_{1}^{\text {per }}(\mathrm{R}) \times I \longrightarrow \operatorname{Diff}_{1}^{\text {per }}(\mathrm{R}),
$$

where $H(g, s)(x)=(1-s) g(x)+s x$ for $x \in \mathrm{R}, s \in I=[0,1]$.

Hence $\pi: \operatorname{Diff}_{1}^{\text {per }}(\mathrm{R}) \longrightarrow \operatorname{Diff}_{+}\left(S^{1}\right)$ is a universal covering space of $\operatorname{Diff}_{+}\left(S^{1}\right)$. Its group of covering transformations is the additive group of integers $\mathrm{Z}$ acting on $\operatorname{Diff}_{1}^{\text {per }}(\mathrm{R})$ by $n(g)(x)=g(x)+n$ for $n \in \mathbf{Z}, x \in \mathrm{R}$.

If a 1-parameter group $\left\{f_{t}\right\}_{t \in \mathrm{R}}$ of diffeomorphisms of $S^{1}$ is given, we can look upon it as a curve in $\operatorname{Diff}_{+}\left(S^{1}\right)$. Then the 1-parameter group $\left\{\tilde{f}_{t}\right\}_{t \in \mathrm{R}}$ of diffeomorphisms of $\mathrm{R}$ constructed above is just the lifting of this curve to $\operatorname{Diff}_{1}^{\text {per }}(\mathrm{R})$ with the starting point $(t=0)$ at id $_{\mathrm{R}}$.

\section{Construction of a deformation}

2.1. Let $R$ be a compact Riemann surface of genus $g \geq 2$. By "Riemann surface" we mean a compact complex manifold of complex dimension 1.

By the Uniformization Theorem $R$ can be described as a quotient of the complex upper half-plane $\mathrm{H}$ by a Fuchsian group $\Gamma$ acting freely and properly discontinuously on $\mathrm{H}, \quad R=\mathrm{H} / \Gamma$. The hyperbolic Poincare metric on $\mathrm{H}$ induces then a Riemannian metric on $R$.

Let $C_{0}$ and $C_{1}$ be two simple closed oriented geodesics on $R$.

Definition 2.1. The pair of geodesics $\left(C_{0}, C_{1}\right)$ is called a 1-pair if $C_{0}$ and $C_{1}$ intersect in exactly one point.

Given a smooth vector field $\mathfrak{X}$ on $S^{1}$, a compact Riemann surface $R$ of genus $g \geq 2$ and a 1-pair of geodesics $\left(C_{0}, C_{1}\right)$ on $R$, we shall construct an infinitesimal deformation of $R$.

If the vector field $\mathfrak{X}$ is constant i.e. if $\mathfrak{X}=a \frac{\widehat{d}}{d x}$ for some constant $a \in \mathrm{R}$, then the resulting deformation does not depend on the choice of $C_{1}$ but only on the geodesic $C_{0}$ and it represents the infinitesimal Fenchel-Nielsen deformation of $R$ along $C_{0}$ (as described in [5]) with the speed depending on $a$. In this sense our construction generalizes the Fenchel-Nielsen deformation.

2.2. Let $\left(C_{0}, C_{1}\right)$ be a 1-pair of geodesics on the Riemann surface $R, R=\mathrm{H} / \Gamma$. There is an element $\gamma_{0} \in \Gamma$ such that $C_{0}$ is the projection to $R$ 
of the axis of $\gamma_{0}$ in $\mathrm{H}$. Conjugating $\Gamma$ with a Möbius transformation if necessary, we can assume that 0 and $\infty$ are the repelling respectively the attracting fixed point of $\gamma_{0}$. It follows that

$$
\gamma_{0}(z)=\lambda z, \quad z \in \mathbf{H},
$$

with $\lambda$ being a real number $>1$. The axis of $\gamma_{0}$ is the positive imaginary halfaxis.

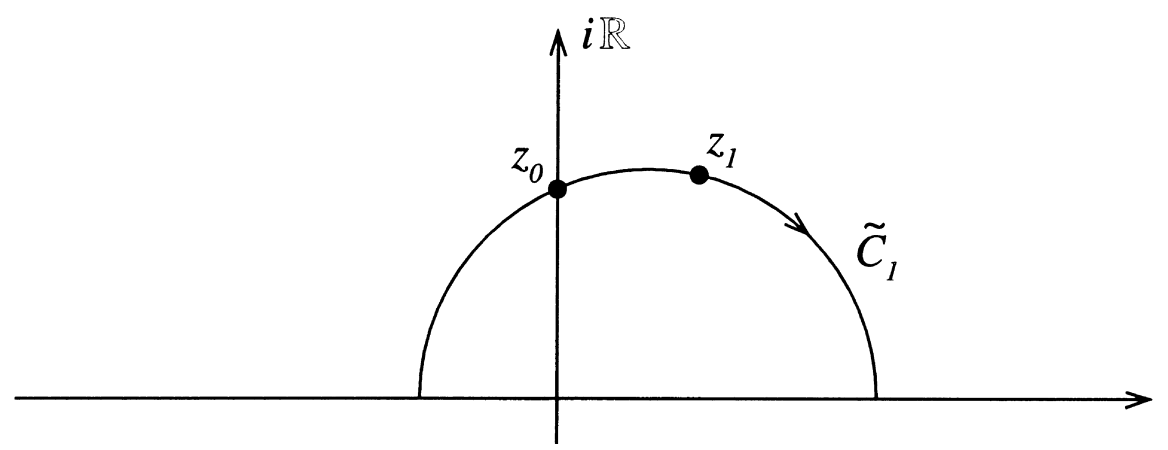

Figure 1.

Let $x_{0} \in R$ be the intersection point of $C_{0}$ and $C_{1}$. Let $C_{1}$ be parametrized by its arc-length, $C_{1}=C_{1}(t)$, in such a way that $x_{0}=C_{1}(0)=C_{1}(q)$, where $q$ is the length of $C_{1}$. Choose a point $z_{0}=s i \in \mathrm{H}, s>0$, lying on the axis of $\gamma_{0}$, which projects to $x_{0}$. Let $\tilde{C}_{1}=\tilde{C}_{1}(t)$ be the lifting of $C_{1}$ to $\mathrm{H}$ with $\tilde{C}_{1}(0)=z_{0}$. $\tilde{C}_{1}$ is a geodesic in $\mathrm{H}$. Let $z_{1}=\tilde{C}_{1}(q) \in \mathrm{H}$. Then $z_{1}$ projects to $x_{0}$ in $R$ and, hence, there is an element $\gamma_{1} \in \Gamma$ such that $z_{1}=\gamma_{1}\left(z_{0}\right)$. It follows that the geodesic $\tilde{C}_{1}$ is the axis of the hyperbolic Möbius transformation $\gamma_{1}$.

By cojugating $\Gamma$ again, if necessary, with a Möbius transformation $\gamma$ of the form $\gamma(z)=\mu z, \mu>0$, we can assume that $s=1$ i.e. that $z_{0}=i \in \mathrm{H}$.

The only role the geodesic $C_{1}$ is playing in our construction is to distinguish a point on the geodesic $C_{0}$ (the point of intersection). This point allows us then to identify $C_{0}$ with the circle $S^{1}$.

This way to distinguish the point on $C_{0}$ depends only on the free homotopy classes of the curves in $R$ represented by $C_{0}$ and $C_{1}$. Therefore, it allows the construction to be performed on the Teichmüller space $T(R)$ of $R$ (see Section 5).

2.3. Let $\left(C_{0}, C_{1}\right)$ be a 1-pair of geodesics on the Riemann surface $R, R=\mathrm{H} / \Gamma$. As explained above there are two elements $\gamma_{0}, \gamma_{1} \in \Gamma$ with the axes $\tilde{C}_{0}$ and $\tilde{C}_{1}$ respectively, such that $\tilde{C}_{i}$ projects to $C_{i}, i=0,1$. Moreover we can assume that $\gamma_{0}(z)=\lambda z, z \in \mathrm{H}$, for some $\lambda>1$ and that the intersection point of $\tilde{C}_{0}$ and $\tilde{C}_{1}$ is $z_{0}=i \in \mathrm{H}$. 
The length of the geodesic $C_{0}$ is equal to $l=\log \lambda$. By the Collar Theorem, [1; Thm 4.1.1, p. 94], there is a real number $\varepsilon=\varepsilon(l), 0<\varepsilon<\pi / 2$, depending only on $l$ such that the sector

$$
\tilde{W}=\left\{\begin{array}{l|l}
z \in \mathrm{H} & \frac{\pi}{2}-\varepsilon<\arg z<\frac{\pi}{2}+\varepsilon
\end{array}\right\}
$$

of the upper half-plane $\mathrm{H}$ projects to a tubular neighbourhood of $C_{0}$ in $R$.

Choose a smooth non-decreasing function $s:[0, \pi] \longrightarrow \mathrm{R}$ such that $s(\theta)=0$ for $\theta \leq \frac{\pi}{2}-\frac{\varepsilon}{2}$ and $s(\theta)=1$ for $\theta \geq \frac{\pi}{2}-\frac{\varepsilon}{4}$.

Let $\mathfrak{X}$ be a smooth vector field on the circle $S^{1}$. $\mathfrak{X}$ generates a 1-parameter group $f_{t}: S^{1} \longrightarrow S^{1}, t \in \mathrm{R}$, of diffeomorphisms of $S^{1}$. As explained in Section 1 , this group lifts to a 1 -parameter group $\tilde{f}_{t}: \mathbf{R} \longrightarrow \mathbf{R}$ of diffeomorphisms of $\mathrm{R}$ satisfying

$$
\tilde{f}_{t}(x+1)=\tilde{f}_{t}(x)+1 \quad \text { for all } x, t \in \mathrm{R} .
$$

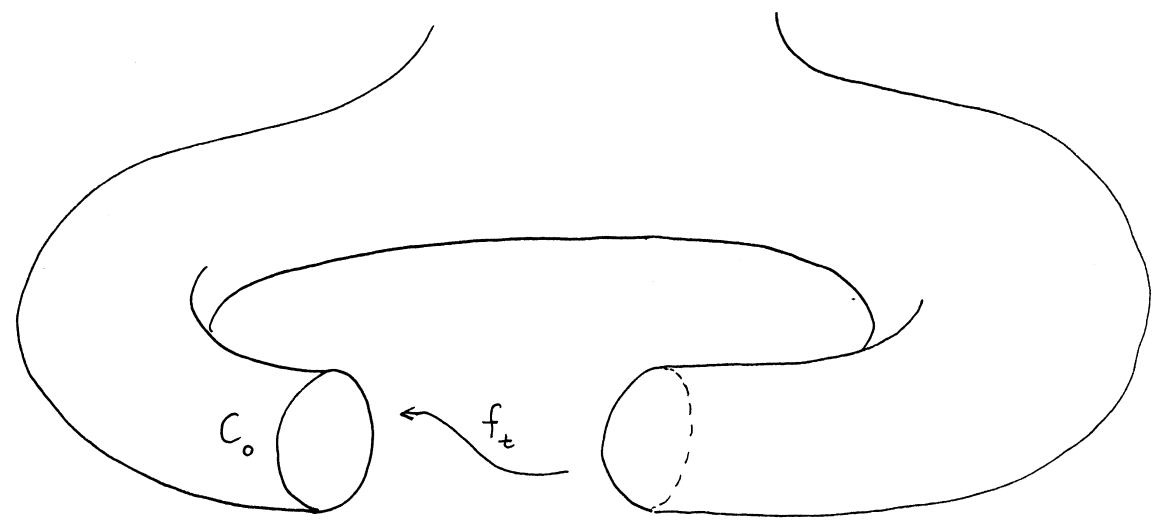

Figure 2 .

The geometric meaning of the deformation which we are going to construct is as follows: cut the surface $R$ along the geodesic $C_{0}$, change the position of one side of the cut by the diffeomorphism $f_{t}$ and then reglue both sides of the cut in their new position.

That, however, requires an identification of $C_{0}$ with the circle $S^{1}$. Such an identification is obtained by identifying the intersection point of $C_{0}$ and $C_{1}$ with $1 \in S^{1}$ and by the standard parametrization of the oriented closed geodesic $C_{0}$. (Observe that as long as only the Fenchel-Nielsen deformation was considered, the identification of the point was not necessary since in that case the diffeomorphisms $f_{t}$ were just rotations of the circle and these are rotation-invariant). 
We shall now describe our construction.

Define a 1-parameter family of functions $\psi_{t}: \mathrm{H} \longrightarrow \mathrm{R}, t \in \mathrm{R}$, by

$$
\psi_{t}(z)=\psi_{t}\left(r e^{i \theta}\right)=l \tilde{f}_{t s(\theta)}\left(\frac{1}{l} \log (r)\right)-\log (r)
$$

for $z=r e^{i \theta} \in \mathrm{H}$.

Then define a 1-parameter family of mappings $F_{t}: \mathrm{H} \longrightarrow \mathrm{H}, \quad t \in \mathrm{R}$, by

$$
F_{t}(z)=e^{\psi_{t}(z)} \cdot z .
$$

Observe that $\arg F_{t}(z)=\arg z$ for all $z \in \mathrm{H}, t \in \mathrm{R}$. It follows then immediately from (2.3) and (2.4) that

$$
F_{t_{1}} \circ F_{t_{2}}=F_{t_{1}+t_{2}} \text { and } F_{0}=\text { id. }
$$

Both $\psi_{t}$ and $F_{t}$ are $C^{\infty}$-functions of variables $t$ and $z$. Hence, for every $t \in \mathrm{R}$, the map $F_{t}$ is a smooth diffeomorphism of $\mathrm{H}$.

Observe also that, because of (2.2), we have

$$
F_{t} \circ \gamma_{0}=\gamma_{0} \circ F_{t}, \quad t \in \mathrm{R} .
$$
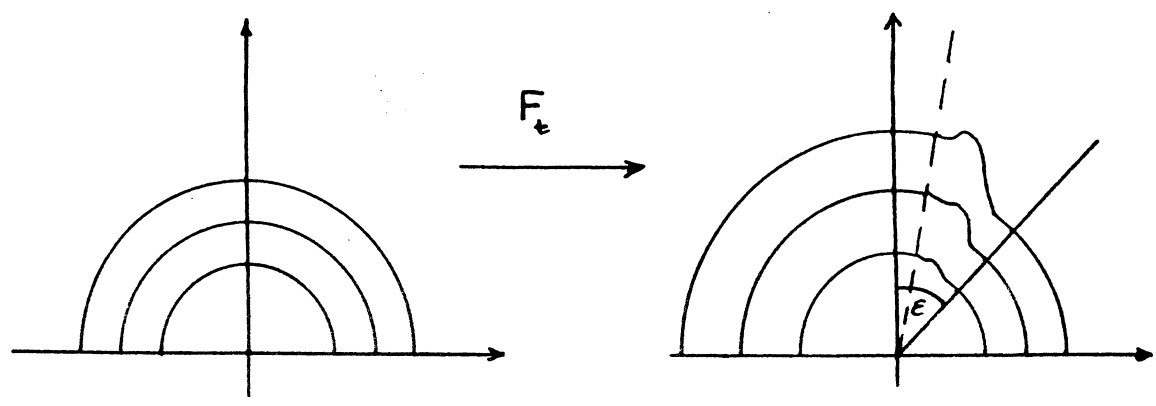

Figure 3.

The geometric meaning of the maps $F_{t}$ is as follows: identify the oriented geodesic $C_{0}$ with the circle $S^{1}$ in the way described above. Then we can look upon the 1-parameter group of diffeomorphisms $f_{t}$ as acting on $C_{0}$. Choose some $t \in \mathrm{R}$. We want to describe the displacement in the collar neighbourhood of $C_{0}$ which starts with the identity on one side of the collar and then gradually maps the consecutive layers of the collar into themselves by the maps $f_{s}$ with varying parameter $s$ until it arrives at the value $s=t$. From that layer on the mapping is done by $f_{t}$ with constant $t$. The map $F_{t}$ describes the lifting of such a displacement to the universal cover $\tilde{W}$ of the collar. Actually, $\tilde{W}$ is a sector in $\mathrm{H}$ and the map $F_{t}$ is extended to the whole upper halfplane $\mathrm{H}$. 
We shall now compute the complex dilatation of $F_{t}$. Using $2 \log r=\log (z \bar{z})$, we get $(\log r)_{z}=\frac{1}{2 z}$ and $(\log r)_{\bar{z}}=\frac{1}{2 \bar{z}}$. Similarily, using $\theta=-i(\log z-\log r)$, we get $(\theta)_{z}=\frac{1}{2 i z}$ and $(\theta)_{\bar{z}}=-\frac{1}{2 i \bar{z}}$. Therefore

$$
\begin{aligned}
\left(\psi_{t}(z)\right)_{z} & =\left(i \tilde{f}_{t s(\theta)}\left(\frac{1}{l} \log (r)\right)-\log (r)\right)_{z} \\
& =l t s^{\prime}(\theta) \frac{1}{2 i z}\left(\frac{d}{d t} \tilde{f}\right)_{t s(\theta)}\left(\frac{1}{l} \log (r)\right)+\frac{1}{2 z}\left(\frac{d}{d x} \tilde{f}\right)_{t s(\theta)}\left(\frac{1}{l} \log (r)\right)-\frac{1}{2 z}
\end{aligned}
$$

and

$$
\begin{aligned}
\left(F_{t}(z)\right)_{z} & =\left(e^{\psi_{t}(z)} \cdot z\right)_{z}=e^{\psi_{t}(z)}\left(1+z\left(\psi_{t}(z)\right)_{z}\right) \\
& =\frac{1}{2} e^{\psi_{t}(z)}\left[1-i l t s^{\prime}(\theta)\left(\frac{d}{d t} \tilde{f}\right)_{t s(\theta)}\left(\frac{1}{l} \log (r)\right)+\left(\frac{d}{d x} \tilde{f}\right)_{t s(\theta)}\left(\frac{1}{l} \log (r)\right)\right] .
\end{aligned}
$$

Similarily we obtain

$$
\begin{aligned}
\left(F_{t}(z)\right)_{\bar{z}} & =\left(e^{\psi_{t}(z)} \cdot z\right)_{\bar{z}}=z e^{\psi_{t}(z)}\left(\psi_{t}(z)\right)_{\bar{z}} \\
& =-\frac{z}{2 \bar{z}} e^{\psi_{t}(z)}\left[1-i l t s^{\prime}(\theta)\left(\frac{d}{d t} \tilde{f}\right)_{t s(\theta)}\left(\frac{1}{l} \log (r)\right)\right. \\
& \left.-\left(\frac{d}{d x} \tilde{f}\right)_{t s(\theta)}\left(\frac{1}{l} \log (r)\right)\right] .
\end{aligned}
$$

Hence the complex dilatation of the mapping $F_{t}: \mathrm{H} \longrightarrow \mathrm{H}$ is

(2.7) $\mu\left(F_{t}\right)(z)=\frac{\left(F_{t}(z)\right)_{\bar{z}}}{\left(F_{t}(z)\right)_{z}}$

$$
=-\frac{z}{\bar{z}}\left[1-\frac{2\left(\frac{d}{d x} \tilde{f}\right)_{t s(\theta)}\left(\frac{1}{l} \log (r)\right)}{1-i l t s^{\prime}(\theta)\left(\frac{d}{d t} \tilde{f}\right)_{t s(\theta)}\left(\frac{1}{l} \log (r)\right)+\left(\frac{d}{d x} \tilde{f}\right)_{t s(\theta)}\left(\frac{1}{l} \log (r)\right)}\right] .
$$

REMARK 2.8. 1) Observe that since $s(\theta)=0$ for $0 \leq \theta \leq \frac{\pi-\varepsilon}{2}$ and since 
$\left(\frac{d}{d x} \tilde{f}\right)_{0}(x) \equiv 1$, then for all vector fields $\mathfrak{X}$ we have $\mu\left(F_{t}\right)(z)=0$ for $z$ such that $0 \leq \arg (z) \leq \frac{\pi-\varepsilon}{2}$.

2) Observe also that for the Fenchel-Nielsen deformation which corresponds to the case when the vector field $\mathfrak{X}=c \frac{\widehat{d}}{d x}, c$-constant, we have $\tilde{f}_{t}(x)=x+c t$. Then $\left(\frac{d}{d x} \tilde{f}\right)_{s}(x) \equiv 1,\left(\frac{d}{d t} \tilde{f}\right)_{s}(x) \equiv c$ and

$$
\mu\left(F_{t}\right)(z)=-\frac{z}{\bar{z}}\left(1-\frac{2}{2-\operatorname{iltcs}^{\prime}(\theta)}\right), \quad z \in \mathrm{H} .
$$

(Compare with [5; p. 503] or [2; p. 220].) Since $s^{\prime}(\theta)=0$ also for $\frac{\pi}{2}-\frac{\varepsilon}{4} \leq \theta \leq \pi$, the Beltrami coefficients $\mu\left(F_{t}\right)$ for the Fenchel-Nielsen deformation are supported in the sector $\frac{\pi}{2}-\frac{\varepsilon}{2} \leq \theta \leq \frac{\pi}{2}-\frac{\varepsilon}{4}$. This is however not the case if we consider more general deformations.

Denote

$$
\begin{aligned}
& a=a(t, z)=\left(\frac{d}{d x} \tilde{f}\right)_{t s(\theta)}\left(\frac{1}{l} \log (r)\right), \\
& b=b(t, z)=l t s^{\prime}(\theta)\left(\frac{d}{d t} \tilde{f}\right)_{t s(\theta)}\left(\frac{1}{l} \log (r)\right) .
\end{aligned}
$$

According to (1.2) and (1.3) there exist real constants $\alpha_{t}^{1}, \alpha_{t}^{2}, B>0$ such that $\alpha_{t}^{1} \leq a \leq \alpha_{t}^{2}$ and $|b| \leq B$ for all $z \in \mathrm{H}$. Then

$$
\begin{aligned}
\left|\mu\left(F_{t}\right)(z)\right| & =\left|\frac{1-a-i b}{1+a-i b}\right|=\left(1-\frac{4 a}{(1+a)^{2}+b^{2}}\right)^{1 / 2} \\
& \leq\left(1-\frac{4 a}{\left(1+a^{2}\right)\left(1+B^{2}\right)}\right)^{1 / 2} \\
& \leq \max _{j=1,2}\left(1-\frac{4 \alpha_{t}^{j}}{\left(1+\alpha_{t}^{j}\right)^{2}\left(1+B^{2}\right)}\right)^{1 / 2} \\
& =k<1,
\end{aligned}
$$


for all $z \in \mathrm{H}$. Therefore, for every $t \in \mathrm{R}, F_{t}: \mathrm{H} \longrightarrow \mathrm{H}$ is a quasiconformal mapping.

Let $\left\langle\gamma_{0}\right\rangle$ be the subgroup of $\Gamma$ generated by the transformation $\gamma_{0}$ and let $B\left(\mathrm{H},\left\langle\gamma_{0}\right\rangle\right)$ be the space of Beltrami differentials on $\mathrm{H}$ with respect to the group $\left\langle\gamma_{0}\right\rangle$ (see [2; p. 124]). Let $B\left(\mathrm{H},\left\langle\gamma_{0}\right\rangle\right)_{1}=\left\{\mu \in B\left(\mathrm{H},\left\langle\gamma_{0}\right\rangle\right) \mid\|\mu\|_{\infty}<1\right\}$ be the corresponding space of Beltrami coefficients.

It follows from (2.6) and (2.9) that

$$
\mu\left(F_{t}\right) \in B\left(\mathrm{H},\left\langle\gamma_{0}\right\rangle\right)_{1}, \quad t \in \mathrm{R} .
$$

Since $F_{0}=\mathrm{id}$, we have $\mu\left(F_{0}\right)=0 .\left\{\mu\left(F_{t}\right) \mid t \in \mathrm{R}\right\}$ is a curve in the space of Beltrami coefficients $B\left(\mathrm{H},\left\langle\gamma_{0}\right\rangle\right)_{1}$. The tangent vector to this curve at $t=0$ is

$$
\begin{aligned}
\left.\frac{\partial}{\partial t} \mu\left(F_{t}\right)\right|_{t=0}(z) & =\frac{z}{2 \bar{z}}\left[s(\theta)\left(\frac{\partial^{2} \tilde{f}}{\partial t \partial x}\right)_{0}\left(\frac{1}{l} \log (r)\right)\right. \\
& \left.+i l s^{\prime}(\theta)\left(\frac{\partial \tilde{f}}{\partial t}\right)_{0}\left(\frac{1}{l} \log (r)\right)\right] \\
& =\frac{z}{2 \bar{z}}\left[s(\theta) \tilde{h}^{\prime}\left(\frac{1}{l} \log (r)\right)+i l s^{\prime}(\theta) \tilde{h}\left(\frac{1}{l} \log (r)\right)\right],
\end{aligned}
$$

where $\tilde{\mathfrak{X}}$ is the lifting to $\mathrm{R}$ of the vector field $\mathfrak{X}$ on $S^{1}$ and the function $\tilde{h}: \mathrm{R} \longrightarrow \mathrm{R}$ is given by

$$
\tilde{\mathfrak{X}}=\tilde{h} \cdot \frac{d}{d x} .
$$

Observe again that the infinitesimal Beltrami differential $\left.\frac{\partial}{\partial t} \mu\left(F_{t}\right)\right|_{t=0}(z)$ vanishes for $z$ with $0<\arg z<(\pi-\varepsilon) / 2$.

The 1-parameter family of deformations of the complex structure of the Riemann surface $R$ which we want to associate with the vector field $\mathfrak{X}$ on $S^{1}$ is obtained by cutting $R$ along the goedesic $C_{0}$, moving one side of the cut by the diffeomorphism $f_{t}$ and then regluing both sides of the cut in the new position.

We shall describe only the infinitesimal deformation of the complex structure of $R$ obtained in this way.

To this end, let us first define a Beltrami differential $\nu_{o}$ on $\mathrm{H}$ by 


$$
\nu_{o}(z)= \begin{cases}\left.\frac{\partial}{\partial t} \mu\left(F_{t}\right)\right|_{t=0}(z) & \text { if } \operatorname{Re}(z) \geq 0 \\ 0 & \text { if } \operatorname{Re}(z)<0\end{cases}
$$

i.e.

$$
\nu_{o}(z)=\frac{z}{2 \bar{z}}\left[s(\theta) \tilde{h}^{\prime}\left(\frac{1}{l} \log (r)\right)+i l s^{\prime}(\theta) \tilde{h}\left(\frac{1}{l} \log (r)\right)\right]
$$

if $z=r e^{i \theta}$ with $0<\theta \leq \pi / 2$ and $\nu_{o}(z)=0$ otherwise.

By our construction $\nu_{o}$ vanishes outside the sector $(\pi-\varepsilon) / 2 \leq \arg z \leq \pi / 2$. Moreover, we have

$$
\nu_{o}\left(\gamma_{o}(z)\right) \frac{\overline{\gamma_{o}^{\prime}(z)}}{\gamma_{o}^{\prime}(z)}=\nu_{o}(\lambda z)=\nu_{o}(z) .
$$

This follows from (2.10) or can be checked directly (recall that $\tilde{h}(x+1)=\tilde{h}(x))$.

Now define a Beltrami differential $\nu(\mathfrak{X})$ on $\mathrm{H}$ by

$$
\nu(\mathfrak{X})(z)=\sum_{\gamma \in\left(\gamma_{0} \backslash \backslash \Gamma\right.} \nu_{o}(\gamma(z)) \frac{\overline{\gamma^{\prime}(z)}}{\gamma^{\prime}(z)}, \quad z \in \mathrm{H} .
$$

Concerning convergence of this series: our choice of $\varepsilon$ garanties that for every $z \in \mathrm{H}$ there is at most one term in the series which does not vanish at $z$.

It follows from our construction that $\nu(\mathfrak{X})$ is a Beltrami differential on $\mathrm{H}$ with respect to $\Gamma, \nu(\mathfrak{X}) \in B(\mathrm{H}, \Gamma)$.

It is the Beltrami differential $\nu(\mathfrak{X})$ which describes our infinitesimal deformation of the complex structure of the Riemann surface $R$ induced by the vector field $\mathfrak{X}$ on $S^{1}$.

To be exact: let $T_{B}(\Gamma)$ be the Teichmüller space of the Fuchsian group $\Gamma$. (For the definitions and notations concerning Teichmüller spaces, see [2; Chap. 6]. We follow the notations used in that book).

Let $\Phi: B(\mathrm{H}, \Gamma)_{1} \longrightarrow T_{B}(\Gamma)$ be the Bers projection, [2; p. 150]. We consider $\nu(\mathfrak{X}) \in B(\mathrm{H}, \Gamma)$ as a tangent vector to $B(\mathrm{H}, \Gamma)_{1}$ at 0 . Then

$$
\varphi_{\left(C_{0}, C_{1}\right)}(\mathfrak{X}):=(d \Phi)_{o}(\nu(\mathfrak{X}))
$$

is a tangent vector to the Teichmüller space $T_{B}(\Gamma)$ at the base point. Every such a vector represents an infinitesimal deformation of the complex structure of $R$. The infinitesimal deformation of $R$ induced by the vector field $\mathfrak{X}$ is, by definition, the one represented by $\varphi_{\left(C_{0}, C_{1}\right)}(\mathfrak{X})$.

Let $A_{2}\left(\mathrm{H}^{*}, \Gamma\right)$ be the space of holomorphic quadratic differentials on the lower half-plane $\mathrm{H}^{*}$ with respect to $\Gamma$. Let $B: T_{B}(\Gamma) \longrightarrow A_{2}\left(\mathrm{H}^{*}, \Gamma\right)$ be the Bers embedding. The Bers embedding identifies the tangent space to $T_{B}(\Gamma)$ 
at the base point with the complex vector space $A_{2}\left(\mathrm{H}^{*}, \Gamma\right)$. We shall now proceed to describe the tangent vector $\varphi_{\left(C_{0}, C_{1}\right)}(\mathfrak{X})$ as an element of $A_{2}\left(\mathrm{H}^{*}, \Gamma\right)$.

\section{Description of the deformations by quadratic differentials}

Let $\mathfrak{X}$ be a smooth vector field on $S^{1}$.

Let $R$ be a compact Riemann surface of genus $g \geq 2$ and suppose that $R=\mathrm{H} / \Gamma$, where $\Gamma$ is a Fuchsian group.

Finally, let $\left(C_{0}, C_{1}\right)$ be a 1-pair of geodesics on $R$ (see Definition 2.1).

In Section 2, given such data, we have constructed a Beltrami differential $\nu=\nu(\mathfrak{X}) \in B(\mathrm{H}, \Gamma)$. We look upon $\nu$ as a tangent vector to the space of Beltrami coefficients $B(\mathrm{H}, \Gamma)_{1}$ at 0 . Let $\Phi: B(\mathrm{H}, \Gamma)_{1} \longrightarrow T_{B}(\Gamma)$ be the Bers projection. Then $\varphi_{\left(C_{0}, C_{1}\right)}(\mathfrak{X})=(d \Phi)_{o}(\nu)$ is a tangent vector to the Teichmüller space $T_{B}(\Gamma)$ at the base point and represents a deformation of the Riemann surface $R$.

The Bers embedding $B: T_{B}(\Gamma) \longrightarrow A_{2}\left(\mathrm{H}^{*}, \Gamma\right)$ gives an identification of $\varphi_{\left(C_{0}, C_{1}\right)}(\mathfrak{X})$ with a quadratic differential on $\mathrm{H}^{*}$ with respect to $\Gamma$. We shall now compute this quadratic differential in case when the vector field $\mathfrak{X}$ has a finite Fourier expansion.

First of all observe that the Beltrami differential $\nu=\nu(\mathfrak{X})$ and, hence, the quadratic differential $\varphi_{\left(C_{0}, C_{1}\right)}(\mathfrak{X})$ depends linearily on $\mathfrak{X}$,

$$
\varphi_{\left(C_{0}, C_{1}\right)}\left(a_{1} \mathfrak{X}_{1}+a_{2} \mathfrak{X}_{2}\right)=a_{1} \varphi_{\left(C_{0}, C_{1}\right)}\left(\mathfrak{X}_{1}\right)+a_{2} \varphi_{\left(C_{0}, C_{1}\right)}\left(\mathfrak{X}_{2}\right),
$$

where $\mathfrak{X}_{1}, \mathfrak{X}_{2}$ are smooth vector fields on $S^{1}$ and $a_{1}, a_{2} \in \mathrm{R}$. This follows immediately from (2.12). Moreover, since $A_{2}\left(\mathrm{H}^{*}, \Gamma\right)$ is a vector space over complex numbers, we can extend the definition of $\varphi_{\left(C_{0}, C_{1}\right)}(\mathfrak{X})$ in an obvious way to the case when $\mathfrak{X}$ is a complexified vector field on $S^{1}$ i.e. when $\mathfrak{X}=h \frac{\widehat{d}}{d x}$ with $h: S^{1} \longrightarrow \mathrm{C}$ being a smooth function. Then (3.1) holds with arbitrary $a_{1}, a_{2} \in \mathrm{C}$ and arbitrary complexified vector fields $\mathfrak{X}_{1}, \mathfrak{X}_{2}$ on $S^{1}$.

According to [2; Thm 6.10, p. 157] the quadratic differential $\varphi_{\left(C_{0}, C_{1}\right)}(\mathfrak{X}) \in$ $A_{2}\left(\mathrm{H}^{*}, \Gamma\right)$ is given by

$$
\begin{aligned}
\varphi_{\left(C_{0}, C_{1}\right)}(\mathfrak{X})(z) & =(d \Phi)_{o}(\nu)(z) \\
& =-\frac{6}{\pi} \iint_{H} \frac{\nu(\zeta)}{(\zeta-z)^{4}} d \xi d \eta
\end{aligned}
$$

for $z \in \mathrm{H}^{*}$. (Here $\zeta=\xi+i \eta$ and the integration is with respect to the Lebesgue measure on $\mathrm{H}_{\text {.) }}$ 
We shall now compute the integral in (3.2) in the case when $\mathfrak{X}=\mathfrak{X}_{n}$ is the complexified vector field on $S^{1}$ such that its lifting to $\mathrm{R}$ is given by

$$
\tilde{\mathfrak{X}}=\tilde{\mathfrak{X}}_{n}=\tilde{h}_{n} \cdot \frac{d}{d x}
$$

with

$$
\tilde{h}_{n}(x)=e^{2 \pi i n x}, \quad x \in \mathbf{R},
$$

$n$ being an integer, $n \in Z$. The result will be given as a Poincaré series.

From now on $\log (z)$ is the branch of $\log$ given by $0 \leq \arg z<2 \pi$.

Lemma 3.3. Let $n$ be an integer, $n \neq 0$, and let $\tilde{h}_{n}(x)=e^{2 \pi i n x}$. Let $\nu_{o} \in$ $B\left(\mathrm{H},\left\langle\gamma_{0}\right\rangle\right)$ be the Beltrami differential defined in (2.12) for the vector field $\tilde{\mathfrak{X}}_{n}=\tilde{h}_{n} \cdot \frac{d}{d x}$. Let

$$
I\left(\nu_{o}\right)(z)=-\frac{6}{\pi} \iint_{\mathrm{H}} \frac{\nu_{o}(\zeta)}{(\zeta-z)^{4}} d \xi d \eta \quad \text { for } z \in \mathrm{H}^{*}
$$

Then

$$
I\left(\nu_{o}\right)(z)=\mathscr{B} \cdot \frac{1}{z^{2}} e^{2 \pi i n \log (z) / l}, \quad z \in \mathrm{H}^{*},
$$

where $\mathscr{B}$ is a constant,

$$
\mathscr{B}=\mathscr{B}(l, n)=2 \pi i n\left(e^{-4 \pi^{2} n / l}-1\right)^{-1} e^{\pi^{2} n / l}\left(1+\frac{4 \pi^{2} n^{2}}{l^{2}}\right) .
$$

Proof. By integrating in the polar coordinates we have

$$
\begin{aligned}
I\left(\nu_{o}\right)(z) & =-\frac{6}{\pi} \int_{t=0}^{\pi / 2} \int_{r=0}^{\infty} \frac{r e^{i 2 t}\left[s(t) \tilde{h}_{n}^{\prime}\left(\frac{1}{l} \log (r)\right)+i l s^{\prime}(t) \tilde{h}_{n}\left(\frac{1}{l} \log (r)\right)\right]}{2\left(r e^{i t}-z\right)^{4}} d r d t \\
& =-\frac{3}{\pi} \int_{0}^{\pi / 2} e^{-i 2 t}\left(s(t) \int_{0}^{\infty} \frac{r \tilde{h}_{n}^{\prime}\left(\frac{1}{l} \log (r)\right)}{\left(r-z e^{-i t}\right)^{4}} d r\right. \\
& \left.+i l s^{\prime}(t) \int_{0}^{\infty} \frac{r \tilde{h}_{n}\left(\frac{1}{l} \log (r)\right)}{\left(r-z e^{-i t}\right)^{4}} d r\right) d t .
\end{aligned}
$$


The integral $I_{1}=\int_{0}^{\infty} \frac{r \tilde{h}_{n}\left(\frac{1}{l} \log (r)\right)}{\left(r-z e^{-i t}\right)^{4}} d r$ is computed by the calculus of residues. We integrate the function $f(\zeta)=\frac{\zeta e^{2 \pi i n \log (\zeta) / l}}{\left(\zeta-z e^{-i t}\right)^{4}}$ along a contour of type

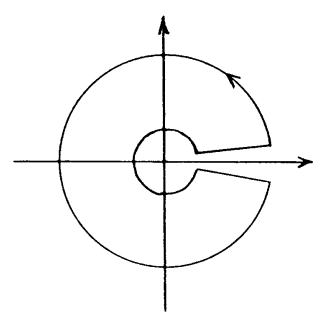

Figure 4.

Observe that the factor $e^{2 \pi i n \log (\zeta) / l}$ is bounded in $\mathrm{C}-\{0\} . f(\zeta)$ has one singularity at $\zeta_{o}=z e^{-i t}$ with a residue

$$
\begin{aligned}
\operatorname{Res}\left(f, \zeta_{o}\right) & =\left.\frac{1}{3 !} \frac{d^{3}}{d \zeta^{3}}\left(\zeta e^{2 \pi i n \log (\zeta) / l}\right)\right|_{\zeta=z e^{-i t}} \\
& =-\frac{\pi i n}{3 l}\left(1+\frac{4 \pi^{2} n^{2}}{l^{2}}\right) \cdot \frac{1}{z^{2}} e^{2 \pi i n \log (z) / l} \cdot e^{(2 \pi n t / l)+i 2 t}
\end{aligned}
$$

Since $\tilde{h}_{n}\left(\frac{\log (r)+2 \pi i}{l}\right)=\tilde{h}_{n}\left(\frac{\log (r)}{l}\right) \cdot e^{-4 \pi^{2} n / l}$, we get

$$
I_{1}=\left(1-e^{-4 \pi^{2} n / l}\right)^{-1} \cdot 2 \pi i \cdot \operatorname{Res}\left(f, \zeta_{o}\right)=\mathscr{A} \cdot e^{(2 \pi n t / l)+i 2 t},
$$

where $\mathscr{A}=\left(1-e^{-4 \pi^{2} n / l}\right)^{-1} \cdot \frac{2 \pi^{2} n}{3 l}\left(1+\frac{4 \pi^{2} n^{2}}{l^{2}}\right) \cdot \frac{1}{z^{2}} e^{2 \pi i n \log (z) / l}$ is independent of $t$.

Since $\tilde{h}_{n}^{\prime}(x)=2 \pi i n \tilde{h}_{n}(x)$, we obtain

$$
\begin{aligned}
I\left(\nu_{o}\right)(z) & =-\frac{3}{\pi} \mathscr{A} \int_{0}^{\pi / 2} e^{-i 2 t}\left(2 \pi i n s(t)+i l s^{\prime}(t)\right) e^{(2 \pi n t / l)+i 2 t} d t \\
& =-\frac{3}{\pi} \mathscr{A} i l \int_{0}^{\pi / 2} \frac{d}{d t}\left(s(t) e^{2 \pi n t / l}\right) d t \\
& =-\frac{3 \mathscr{A} i l}{\pi} \cdot e^{\pi^{2} n / l}
\end{aligned}
$$

which gives the result of Lemma 3.3. 
In the case $n=0$ (which gives the Fenchel-Nielsen deformation) we have

Lemma 3.4. Let $\nu_{o} \in B\left(\mathrm{H},\left\langle\gamma_{0}\right\rangle\right)$ be the Beltrami differential defined in (2.12) for the vector field $\tilde{\mathfrak{X}}_{0}=\frac{d}{d x}$ (i.e. $\left.\tilde{h}(x) \equiv 1\right)$. Let

$$
I\left(\nu_{o}\right)(z)=-\frac{6}{\pi} \iint_{\mathrm{H}} \frac{\nu_{o}(\zeta)}{(\zeta-z)^{4}} d \xi d \eta \quad \text { for } z \in \mathrm{H}^{*} .
$$

Then

$$
I\left(\nu_{o}\right)(z)=-\frac{i l}{2 \pi} \cdot \frac{1}{z^{2}}, \quad z \in \mathrm{H}^{*} .
$$

Proof. By integrating in polar coordinates (since $\tilde{h}^{\prime} \equiv 0$ ):

$$
\begin{aligned}
I\left(\nu_{o}\right)(z) & =-\frac{6}{\pi} \int_{0}^{\pi / 2} \frac{1}{2} e^{i 2 t} i l s^{\prime}(t)\left(\int_{0}^{\infty} \frac{r}{\left(r e^{i t}-z\right)^{4}} d r\right) d t \\
& =-\frac{3 i l}{\pi} \int_{0}^{\pi / 2} e^{i 2 t} s^{\prime}(t) \cdot \frac{1}{6 e^{i 2 t} z^{2}} d t=-\frac{i l}{2 \pi z^{2}} \int_{0}^{\pi / 2} s^{\prime}(t) d t \\
& =-\frac{i l}{2 \pi} \cdot \frac{1}{z^{2}} .
\end{aligned}
$$

Let us recall that the Bergman kernel for the upper half-plane $\mathscr{K}_{\mathrm{H}}(z, \zeta)=\frac{12}{\pi} \cdot \frac{1}{(\zeta-z)^{4}}, \zeta \in \mathrm{H}, z \in \mathrm{H}^{*}$, has the following invariance property:

$$
\mathscr{K}_{\mathrm{H}}(z, \zeta)=\mathscr{K}_{\mathrm{H}}(\gamma(z), \gamma(\zeta)) \cdot \gamma^{\prime}(z)^{2} \cdot \gamma^{\prime}(\zeta)^{2}, \quad \zeta \in \mathrm{H}, z \in \mathrm{H}^{*},
$$

for all $\gamma \in \operatorname{PSL}(2, \mathbf{R})$.

It follows that 


$$
\begin{aligned}
-\frac{6}{\pi} \iint_{\mathrm{H}} \frac{\nu_{o}(\gamma(\zeta)) \overline{\gamma^{\prime}(\zeta)}}{(\zeta-z)^{4}} d \xi d \eta & =-\frac{6}{\pi} \iint_{\mathrm{H}} \frac{\nu_{o}(\gamma(\zeta))\left|\gamma^{\prime}(\zeta)\right|^{2}}{(\zeta-z)^{4} \gamma^{\prime}(\zeta)^{2}} d \xi d \eta \\
& =-\frac{6}{\pi} \iint_{\mathrm{H}} \frac{\nu_{o}(\gamma(\zeta))\left|\gamma^{\prime}(\zeta)\right|^{2}}{(\gamma(\zeta)-\gamma(z))^{4}} \cdot \gamma^{\prime}(z)^{2} d \xi d \eta \\
& =-\frac{6}{\pi} \gamma^{\prime}(z)^{2} \iint_{\mathrm{H}} \frac{\nu_{o}(\zeta)}{(\zeta-\gamma(z))^{4}} d \xi d \eta \\
& =I\left(\nu_{o}\right)(\gamma(z)) \cdot \gamma^{\prime}(z)^{2}
\end{aligned}
$$

Since all the partial sums of the Beltrami differential

$$
\nu(z)=\sum_{\gamma \in\left\langle\gamma_{0}\right\rangle \backslash \Gamma} \nu_{o}(\gamma(z)) \overline{\frac{\gamma^{\prime}(z)}{\gamma^{\prime}(z)}}
$$

are bounded by $\|\nu\|_{\infty}$ and since for every $z \in \mathrm{H}^{*}$ the function $g(\zeta)=\frac{1}{(\zeta-z)^{4}}$ is absolutly integrable in $\mathrm{H}$, it follows from (3.2) and (3.5) that

$$
\begin{aligned}
\varphi_{\left(C_{0}, C_{1}\right)}(\mathfrak{X})(z) & =(d \Phi)_{o}(\nu)(z)=-\frac{6}{\pi} \iint_{\mathrm{H}} \frac{\nu(\zeta)}{(\zeta-z)^{4}} d \xi d \eta \\
& =\sum_{\gamma \in\left\langle\gamma_{0}\right\rangle \backslash \Gamma}-\frac{6}{\pi} \iint_{\mathrm{H}} \frac{\nu_{o}(\gamma(\zeta)) \frac{\overline{\gamma^{\prime}(\zeta)}}{\gamma^{\prime}(\zeta)}}{(\zeta-z)^{4}} d \xi d \eta \\
& =\sum_{\gamma \in\left\langle\gamma_{0}\right\rangle \backslash \Gamma} I\left(\nu_{o}\right)(\gamma(z)) \cdot \gamma^{\prime}(z)^{2} .
\end{aligned}
$$

Finally, we have

THEOREM 3.7. If $n$ is an integer and the complexified vector field $\mathfrak{X}_{n}$ on $S^{1}$ is given by the function $\tilde{h}_{n}: \mathrm{R} \longrightarrow \mathrm{C}, \tilde{h}_{n}(x)=e^{2 \pi i n x}$, then the quadratic differential $\varphi_{\left(C_{0}, C_{1}\right)}\left(\mathfrak{X}_{n}\right) \in A_{2}\left(\mathrm{H}^{*}, \Gamma\right)$ is given by the Poincaré series

$$
\varphi_{\left(C_{0}, C_{1}\right)}\left(\mathfrak{X}_{n}\right)=\mathscr{B}_{n} \sum_{\gamma \in\left\langle\gamma_{0}\right\rangle \backslash \Gamma} e^{2 \pi i n \log (\gamma) / l}\left(\frac{\gamma^{\prime}}{\gamma}\right)^{2},
$$

with $\mathscr{B}_{n}$ being a constant, 


$$
\mathscr{B}_{n}=\mathscr{B}(n, l)= \begin{cases}-\frac{i l}{2 \pi} & \text { if } n=0, \\ 2 \pi i n e^{\pi^{2} n / l}\left(1+\frac{4 \pi^{2} n^{2}}{l^{2}}\right)\left(e^{-4 \pi^{2} n / l}-1\right)^{-1} & \text { if } n \neq 0,\end{cases}
$$

and the series converges absolutely and locally uniformly in $\mathrm{H}^{*}$.

Proof. The formula for $\varphi_{\left(C_{0}, C_{1}\right)}\left(\mathfrak{X}_{n}\right)$ follows from (3.6) together with Lemmas 3.3 and 3.4. For the statement about convergence: since $\pi<\operatorname{Im} \log (z)<2 \pi$ for $z \in \mathrm{H}^{*}$ so for any given $n$ the function $e^{2 \pi i n \log (z) / l}$ is bounded in $\mathrm{H}^{*}$. Hence the claim about convergence follows from [2; Thm 7.2, p. 186].

Remarks 3.8. 1) Theorem 3.7 generalizes a result of S. Wolpert, [5; Thm 2.7, p. 516], [2; Thm 8.2, p. 223], describing the Poincaré series corresponding to the Fenchel-Nielsen deformation. It is the case $n=0$ of our Theorem 3.7. Observe that compared to Wolpert's original version, [5], our constant $\mathscr{B}_{0}$ has an extra factor $l$, the length of the geodesic $C_{0}$. This is due to the fact that our deformations are done by identifying $C_{0}$ with the circle $S^{1}$ and, hence, when looked upon in the Riemann surface $R$ these deformations are done with speed $l$ while Wolpert's deformation was done with speed 1 . There is also a difference of sign when compared to [2]. This is because the Fenchel-Nielsen deformation in [2] is done in opposite direction when compared to Wolpert's one and ours.

2) It follows from Theorem 3.7 that the infinitesimal deformations associated to the vector fields $\mathfrak{X}_{n}$ are independent of the choice of the auxiliary function $s(\theta)$ and depend only on $n$, the Riemann surface $R$ and the 1-pair of geodesics $\left(C_{0}, C_{1}\right)$. Consequently, the same holds for any vector field $\mathfrak{X}$ with finite Fourier expansion - the infinitesimal deformation depends only on $\mathfrak{X}, R$ and $\left(C_{0}, C_{1}\right)$. Actually, this can be proven for any $C^{\infty}$ vector field $\mathfrak{X}$ on $S^{1}$. That will be shown in another paper.

3) In Section 4 we shall give another, independent proof of Theorem 3.7 closer to the one given in [5] for the Fenchel-Nielsen deformation. This second proof is somewhat more complicated, but it gives at the same time a description of the Eichler cohomology classes corresponding to the deformations induced by the vector fields $\mathfrak{X}_{n}$.

\section{Description of the deformations by Eichler cohomology classes}

Let $\nu=\nu(\mathfrak{X})$ be the Beltrami differential on $\mathrm{H}$ defined in (2.14), $\nu \in B(\mathrm{H}, \Gamma)$. $\nu$ determines an infinitesimal deformation $\varphi_{\left(C_{0}, C_{1}\right)}(\mathfrak{X})$ of the Riemann surface $R=\mathrm{H} / \Gamma$. In Section 3, assuming that $\mathfrak{X}$ had a finite Fourier expansion, we have given a description of $\varphi_{\left(C_{0}, C_{1}\right)}(\mathfrak{X})$ as a Poincaré series. In this Section we 
shall describe the Eichler cohomology class corresponding to $\varphi_{\left(C_{0}, C_{1}\right)}(\mathfrak{X})$ (again for $\mathfrak{X}$ with finite Fourier expansion ).

We extend $\nu$ to a Beltrami differential $\hat{\nu}$ on $\mathrm{C}$ by

$$
\hat{\nu}(z)= \begin{cases}\nu(z), & z \in \mathbf{H}, \\ 0, & z \in \mathbf{C}-\mathbf{H} .\end{cases}
$$

Let us consider a potential function $F_{\nu}: \mathrm{C} \longrightarrow \mathrm{C}$ for $\hat{\nu}$ given by

$$
F_{\nu}(z)=-\frac{z(z-1)}{\pi} \iint_{\mathrm{H}} \frac{\nu(\zeta)}{\zeta(\zeta-1)(\zeta-z)} d \xi d \eta,
$$

(see [2; p. 197] and [3; Chap. IV, Lemma 1.4, p. 136]).

Let $\Pi_{2}$ be the space of polynomials in one complex variable of degree $\leq 2$. The group $\Gamma$ acts on $\Pi_{2}$ via

$$
\gamma_{*}(P)=\frac{P \circ \gamma}{\gamma^{\prime}}, \quad \gamma \in \Gamma, P \in \Pi_{2} .
$$

The space of infinitesimal deformations of $R$ is identified with a subspace of the first Eichler cohomology group $H^{1}\left(\Gamma, \Pi_{2}\right)$.

The Eichler cohomology class $\left[\chi_{\nu}\right] \in H^{1}\left(\Gamma, \Pi_{2}\right)$ corresponding to the infinitesimal deformation $\varphi_{\left(C_{0}, C_{1}\right)}(\mathfrak{X})$ is given by the cocycle

$$
\chi_{\nu}: \Gamma \longrightarrow \Pi_{2},
$$

where

$$
\chi_{\nu}(\gamma)=\frac{F_{\nu} \circ \gamma}{\gamma^{\prime}}-F_{\nu}
$$

(See [2; p. 197].)

We shall now determine the cocycle $\chi_{\nu}$.

Let $n$ be an integer, $n \in \mathbb{Z}$, and let $\mathfrak{X}=\mathfrak{X}_{n}$ be the (complexified) vector field on $S^{1}$ given by the function $\tilde{h}_{n}(x)=e^{2 \pi i n x}, \tilde{\mathfrak{x}}_{n}=\tilde{h}_{n} \cdot \frac{d}{d x}$. Let $\nu_{o}^{n} \in B\left(\mathrm{H},\left\langle\gamma_{0}\right\rangle\right)$ be the Beltrami differential defined in (2.12) for $\tilde{h}=\tilde{h}_{n}$. Finally, let $I_{n}(z)$ be the potential function for $\nu_{o}^{n}$,

$$
I_{n}(z)=-\frac{z(z-1)}{\pi} \iint_{\mathrm{H}} \frac{\nu_{o}^{n}(\zeta)}{\zeta(\zeta-1)(\zeta-z)} d \xi d \eta, \quad z \in \mathrm{C} .
$$

Lemma 4.4. If $n \neq 0$ then for all $z \in \mathrm{C}-\{0\}$ satisfying $\frac{\pi}{2} \leq \arg z<2 \pi$ one has 


$$
I_{n}(z)=C_{n} z\left(e^{2 \pi i n(\log (z)-2 \pi i) / l}-1\right)
$$

where $C_{n}$ is a constant,

$$
C_{n}=C(n, l)=l e^{\pi^{2} n / l} \cdot\left(e^{4 \pi^{2} n / l}-1\right)^{-1} .
$$

Proof. The proof is very similar to that of Lemma 3.3. Since

$$
\nu_{o}^{n}(z)=\frac{z}{2 \bar{z}}\left[s(\theta) \tilde{h}_{n}^{\prime}\left(\frac{1}{l} \log (r)\right)+i l s^{\prime}(\theta) \tilde{h}_{n}\left(\frac{1}{l} \log (r)\right)\right]
$$

if $z=r e^{i \theta}$ with $0<\theta \leq \frac{\pi}{2}$ and $\nu_{o}^{n}(z)=0$ otherwise, integrating in polar coordinates we obtain

$$
\begin{aligned}
I_{n}(z) & =-\frac{z(z-1)}{2 \pi} \int_{t=0}^{\pi / 2} \int_{r=0}^{\infty} \frac{s(t) \tilde{h}_{n}^{\prime}\left(\frac{1}{l} \log (r)\right)+i l s^{\prime}(t) \tilde{h}_{n}\left(\frac{1}{l} \log (r)\right)}{e^{-i t}\left(r e^{i t}-1\right)\left(r e^{i t}-z\right)} d r d t \\
& =-\frac{z(z-1)}{2 \pi} \int_{t=0}^{\pi / 2} e^{-i t}\left[s(t) \int_{r=0}^{\infty} \frac{\tilde{h}_{n}^{\prime}\left(\frac{1}{l} \log (r)\right)}{\left(r-e^{-i t}\right)\left(r-z e^{-i t}\right)} d r\right. \\
& \left.+i l s^{\prime}(t) \int_{r=0}^{\infty} \frac{\tilde{h}_{n}\left(\frac{1}{l} \log (r)\right)}{\left(r-e^{-i t}\right)\left(r-z e^{-i t}\right)} d r\right] d t .
\end{aligned}
$$

The integral $J_{n}(z, t)=\int_{r=0}^{\infty} \frac{\tilde{h}_{n}\left(\frac{1}{l} \log (r)\right)}{\left(r-e^{-i t}\right)\left(r-z e^{-i t}\right)} d r$ is evaluated by the calculus of residues. We integrate the function $g(\zeta)=\frac{e^{2 \pi i n \log (\zeta) / l}}{\left(\zeta-e^{-i t}\right)\left(\zeta-z e^{-i t}\right)}$ along a contour of type

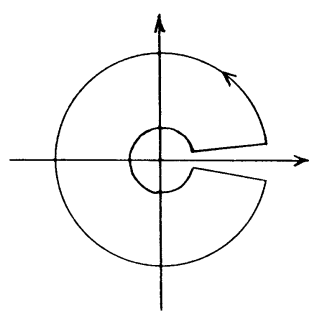

Figure 5. 
Observe that we are interested in the case when $z \neq 0,1$ and that the nominator of $g(\zeta)$ is bounded in $\mathrm{C}-\{0\}$. The function $g(\zeta)$ has two singular points (both simple poles): one at $\zeta_{1}=e^{-i t}$ with a residue

$$
\operatorname{Res}\left(g, \zeta_{1}\right)=e^{i t} \cdot \frac{e^{-2 \pi n(2 \pi-t) / l}}{1-z}
$$

and one at $\zeta_{2}=z e^{-i t}$ with a residue

$$
\operatorname{Res}\left(g, \zeta_{2}\right)=e^{i t} \cdot \frac{e^{2 \pi i n \log \left(z e^{-i t}\right) / l}}{z-1} .
$$

Since $\tilde{h}_{n}((2 \pi i+\log (r)) / l)=\tilde{h}_{n}(\log (r) / l) \cdot e^{-4 \pi^{2} n / l}$, we get

$$
\begin{aligned}
J_{n}(z, t) & =\left(1-e^{-4 \pi^{2} n / l}\right)^{-1} 2 \pi i\left(\operatorname{Res}\left(g, \zeta_{1}\right)+\operatorname{Res}\left(g, \zeta_{2}\right)\right) \\
& =2 \pi i\left(1-e^{-4 \pi^{2} n / l}\right)^{-1} \cdot \frac{e^{i t}}{z-1} \cdot\left(e^{2 \pi i n \log \left(z e^{-i t}\right) / l}-e^{-2 \pi n(2 \pi-t) / l}\right) .
\end{aligned}
$$

If $\frac{\pi}{2} \leq \arg z<2 \pi$ then $\log \left(z e^{-i t}\right)=\log (z)-i$ for all $0 \leq t \leq \frac{\pi}{2}$ and therefore

$$
J_{n}(z, t)=2 \pi i\left(1-e^{-4 \pi^{2} n / l}\right)^{-1} e^{-4 \pi^{2} n / l} \frac{e^{i t}}{z-1} \cdot e^{2 \pi n t / l} \cdot\left(e^{2 \pi i n(\log (z)-2 \pi i) / l}-1\right) .
$$

Let $\mathscr{C}(z)=i\left(e^{4 \pi^{2} n / l}-1\right)^{-1} z\left(e^{2 \pi i n(\log (z)-2 \pi i) / l}-1\right)$. Then

$$
\begin{aligned}
I_{n}(z) & =-\mathscr{C}(z) \int_{t=0}^{\pi / 2} e^{2 \pi n t / l}\left(2 \pi i n s(t)+i l s^{\prime}(t)\right) d t \\
& =-i l \mathscr{C}(z)\left[e^{2 \pi n t / l} s(t)\right]_{t=0}^{t=\pi / 2} \\
& =-i l \mathscr{C}(z) e^{\pi^{2} n / l}
\end{aligned}
$$

which proves Lemma 4.4 .

For the sake of completeness let us also consider the case $n=0$. This has been solved by Wolpert in [5; Sec. 2].

$$
\begin{aligned}
& \text { LEMма } 4.5 . \quad I_{0}(z)=\frac{i l}{2 \pi} z(\log (z)-2 \pi i) \text { for all } z \in \mathrm{C}-\{0\} \text { satisfying } \\
& \frac{\pi}{2} \leq \arg (z)<2 \pi \text {. }
\end{aligned}
$$

Proof. By integrating in the polar coordinates again we get 


$$
\begin{aligned}
I_{0}(z) & =-\frac{z(z-1)}{2 \pi} \int_{t=0}^{\pi / 2} \int_{r=0}^{\infty} \frac{i l s^{\prime}(t)}{e^{-i t}\left(r e^{i t}-1\right)\left(r e^{i t}-z\right)} d r d t \\
& =-\frac{z(z-1) i l}{2 \pi} \int_{t=0}^{\pi / 2} s^{\prime}(t) e^{-i t}\left(\int_{r=0}^{\infty} \frac{d r}{\left(r-e^{-i t}\right)\left(r-z e^{-i t}\right)}\right) d t \\
& =-\frac{z(z-1) i l}{2 \pi} \int_{t=0}^{\pi / 2} s^{\prime}(t) \cdot \frac{1}{z-1}(2 \pi i-\log (z)) d t .
\end{aligned}
$$

The last equality holds provided $\frac{\pi}{2} \leq \arg (z)<2 \pi$. Hence

$$
\begin{aligned}
I_{0}(z) & =-\frac{z i l}{2 \pi}(2 \pi i-\log (z))[s(t)]_{t=0}^{t=\pi / 2} \\
& =\frac{i l}{2 \pi} z(\log (z)-2 \pi i) .
\end{aligned}
$$

Let us now recall that if $\mu$ is any bounded measurable function on $\mathrm{C}$ and if $F_{\mu}(z)$ is defined by

$$
F_{\mu}(z)=-\frac{z(z-1)}{\pi} \iint_{C} \frac{\mu(\zeta)}{\zeta(\zeta-1)(\zeta-z)} d \xi d \eta
$$

then

$$
\begin{aligned}
& \text { (i) } F_{\mu} \text { is a continuous function on } \mathrm{C} \text {, } \\
& \text { (ii) } F_{\mu}(0)=F_{\mu}(1)=0, \\
& \text { (iii) }\left(F_{\mu}\right)_{\bar{z}}=\mu \text { in the sense of distributions, } \\
& \text { (iv) } F_{\mu}(z)=o\left(|z|^{2}\right) \text { as } z \longrightarrow \infty \text {. }
\end{aligned}
$$

See [3; Lemma 1.4, p. 136].

Applying (4.6) (iii) to $I_{n}(z)$ we see that $\left(I_{n}\right)_{\bar{z}}=\nu_{o}^{n}$ in the sense of distributions and, hence,

$$
\left(I_{n} \circ \gamma\right)_{\bar{z}}=\left(\nu_{o}^{n} \circ \gamma\right) \cdot \overline{\gamma^{\prime}}, \quad \gamma \in \Gamma .
$$

It follows that for every $\gamma \in \Gamma$

$$
P_{\gamma}^{n}(z)=\frac{\left(I_{n} \circ \gamma\right)(z)}{\gamma^{\prime}(z)}-\left(-\frac{z(z-1)}{\pi} \iint_{\mathrm{H}} \frac{\nu_{o}^{n}(\gamma(\zeta)) \overline{\gamma^{\prime}(\zeta)}}{\zeta(\zeta-1)(\zeta-z) \gamma^{\prime}(\zeta)} d \xi d \eta\right)
$$

is a holomorphic function on $\mathrm{C}$ and $P_{\gamma}^{n}(z)=O\left(|z|^{2}\right)$ as $z \longrightarrow \infty$. Therefore $P_{\gamma}^{n}(z)$ is a polynomial in $z$ of degree at most 2. Observe that $P_{e}^{n}(z) \equiv 0$, where $e$ is the identity element of $\Gamma$. 
Let $C_{n}=C(n, l), n \neq 0$, be the constant of Lemma 4.4 and let $\log (z)$ be the branch of logarithm given by $-\pi \leq \arg (z)<\pi$.

LEMMA 4.8. Let $n$ be an integer, $n \neq 0$.

(i) If $\gamma_{1}, \gamma_{2} \in \Gamma$ represent the same coset in $\left\langle\gamma_{0}\right\rangle \backslash \Gamma$ then $P_{\gamma_{1}}^{n}=P_{\gamma_{2}}^{n}$.

(ii) If $\gamma \in\left\langle\gamma_{0}\right\rangle$ then $P_{\gamma}^{n}=0$.

(iii) If $\gamma \in \Gamma-\left\langle\gamma_{0}\right\rangle$ and $\gamma(z)=\frac{a z+b}{c z+d}$, ad $-b c=1$, then $P_{\gamma}^{n}(z)=a_{0 \gamma}^{n}+$ $a_{1 \gamma}^{n} z+a_{2 \gamma}^{n} z^{2}$ with

$$
\begin{array}{ll}
a_{2 \gamma}^{n}=a c\left(e^{2 \pi i n \log \left(\frac{a}{c}\right) / l}-1\right) \cdot C_{n}, & \\
a_{0 \gamma}^{n}=b d\left(e^{2 \pi i n \log \left(\frac{b}{d}\right) / l}-1\right) \cdot C_{n} & \text { and with } \\
P_{\gamma}^{n}(1)=(a+b)(c+d)\left(e^{2 \pi i n \log \left(\frac{a+b}{c+d}\right) / l}-1\right) \cdot C_{n} . &
\end{array}
$$

REMARK. The expressions in (iii) are well defined: if $\gamma \in \Gamma-\left\langle\gamma_{0}\right\rangle$ and $\gamma(z)=\frac{a z+b}{c z+d}$ then $a, b, c, d, a+b, c+d \neq 0$, see [5; p. 514]

Proof. (i) It follows from Lemma 4.4 that $\left(I_{n} \circ \gamma_{o}\right)(z)=I_{n}(\lambda z)=\lambda I_{n}(z)$ for all $z \in \mathrm{H}^{*}, n \neq 0$. Hence

$$
\frac{\left(I_{n} \circ\left(\gamma_{o} \circ \gamma\right)\right)(z)}{\left(\gamma_{o} \circ \gamma\right)^{\prime}(z)}=\frac{\left(I_{n} \circ \gamma\right)(z)}{\gamma^{\prime}(z)}, \quad z \in \mathrm{H}^{*}, n \neq 0 .
$$

The integrand in the second term on the right hand side of (4.7) will remain unchanged if $\gamma$ is replaced by $\gamma_{o} \circ \gamma$ since, by $(2.13), \nu_{o}^{n}\left(\gamma_{o}(\zeta)\right)=\nu_{o}^{n}(\zeta), \zeta \in \mathrm{H}$. Thus

$$
P_{\gamma_{\circ} \circ \gamma}^{n}=P_{\gamma}^{n}, \quad \gamma \in \Gamma, n \in \mathbf{Z}, n \neq 0 .
$$

(ii) If $\gamma \in\left\langle\gamma_{0}\right\rangle$ then, by (i), $P_{\gamma}^{n}=P_{e}^{n}=0$.

(iii) Suppose that $\gamma \in \Gamma-\left\langle\gamma_{0}\right\rangle$ and $\gamma(z)=\frac{a z+b}{c z+d}$, $a d-b c=1$. Let

$$
G_{\gamma}^{n}(z)=-\frac{z(z-1)}{\pi} \iint_{\mathrm{H}} \frac{\nu_{o}^{n}(\gamma(\zeta)) \overline{\gamma^{\prime}(\zeta)}}{\zeta(\zeta-1)(\zeta-z) \gamma^{\prime}(\zeta)} d \xi d \eta
$$

According to (4.6), $G_{\gamma}^{n}$ is continuous in $\mathrm{C}, G_{\gamma}^{n}(0)=G_{\gamma}^{n}(1)=0$ and $G_{\gamma}^{n}(z)=o\left(|z|^{2}\right)$ as $z \longrightarrow \infty$. On the other hand, from Lemma 4.4

$$
\begin{aligned}
\frac{I_{n}(\gamma(z))}{\gamma^{\prime}(z)} & =C_{n} \frac{\gamma(z)}{\gamma^{\prime}(z)}\left(e^{2 \pi i n(\log (\gamma(z))-2 \pi i) / l}-1\right) \\
& =C_{n}(a z+b)(c z+d)\left(e^{2 \pi i n\left(\log \left(\frac{a z+b}{c z+d}\right)-2 \pi i\right) / l}-1\right), \quad z \in \mathbf{H}^{*}
\end{aligned}
$$


Hence

$$
\begin{aligned}
& P_{\gamma}^{n}(0)=\lim _{\substack{z \rightarrow 0 \\
z \in \mathrm{H}^{*}}} P_{\gamma}^{n}(z)=\lim _{\substack{z \rightarrow 0 \\
z \in \mathrm{H}^{*}}} \frac{I_{n}(\gamma(z))}{\gamma^{\prime}(z)}=C_{n} b d\left(e^{2 \pi i n \log \left(\frac{b}{d}\right) / l}-1\right), \\
& P_{\gamma}^{n}(1)=\lim _{\substack{z \rightarrow 1 \\
z \in \mathrm{H}^{*}}} \frac{I_{n}(\gamma(z))}{\gamma^{\prime}(z)}=C_{n}(a+b)(c+d)\left(e^{2 \pi i n \log \left(\frac{a+b}{c+d}\right) / l}-1\right),
\end{aligned}
$$

and

$$
\lim _{z \rightarrow \infty} \frac{1}{z^{2}} P_{\gamma}^{n}(z)=\lim _{\substack{z \rightarrow \infty \\ z \in \mathrm{H}^{*}}} \frac{I_{n}(\gamma(z))}{\gamma^{\prime}(z)}=C_{n} a c\left(e^{2 \pi i n \log \left(\frac{a}{c}\right) / l}-1\right) .
$$

This proves part (iii) of Lemma 4.8 .

Again, for the sake of completeness let us recall the corresponding result for $n=0$ from [5; p. 513-514].

Let $C_{0}=\frac{i l}{2 \pi}$.

Lemma 4.9.

(i) For every $\gamma \in \Gamma$

$$
P_{\gamma_{o} \circ \gamma}^{0}(z)=P_{\gamma}^{0}(z)+\frac{i l^{2}}{2 \pi} \cdot \frac{\gamma(z)}{\gamma^{\prime}(z)}, \quad z \in \mathrm{C} .
$$

(ii) If $\gamma=\left(\gamma_{o}\right)^{m}, m \in \mathbf{Z}$, then $P_{\gamma}^{0}(z)=m \frac{i l^{2}}{2 \pi} z$.

(iii) If $\gamma \in \Gamma-\left\langle\gamma_{0}\right\rangle$ and $\gamma(z)=\frac{a z+b}{c z+d}, a d-b c=1$, then $P_{\gamma}^{0}(z)=a_{0 \gamma}^{0}+$ $a_{1 \gamma}^{0} z+a_{2 \gamma}^{0} z^{2}$ with

$$
\begin{aligned}
& a_{2 \gamma}^{0}=a c \log \left(\frac{a}{c}\right) \cdot C_{0}, \\
& a_{0 \gamma}^{0}=b d \log \left(\frac{b}{d}\right) \cdot C_{0} \quad \text { and } \\
& P_{\gamma}^{0}(1)=(a+b)(c+d) \log \left(\frac{a+b}{c+d}\right) \cdot C_{0} .
\end{aligned}
$$

Proof. (i) From the explicit description of $I_{0}(z)$ in Lemma 4.5 one has

$$
\frac{I_{0}\left(\gamma_{o}(z)\right)}{\gamma_{o}^{\prime}(z)}=I_{0}(z)+\frac{i l^{2}}{2 \pi} z
$$

Hence 


$$
\frac{I_{0}\left(\left(\gamma_{o} \circ \gamma\right)(z)\right)}{\left(\gamma_{o} \circ \gamma\right)^{\prime}(z)}=\frac{I_{0}(\gamma(z))}{\gamma^{\prime}(z)}+\frac{i l^{2} \gamma(z)}{2 \pi \gamma^{\prime}(z)}, \quad \gamma \in \Gamma \text {. }
$$

Since the second term on the right hand side of (4.7) is unchanged when one replaces $\gamma$ with $\gamma_{o} \circ \gamma$ (see the proof of Lemma 4.8), we obtain (i).

(ii) follows directly from (i).

(iii) is proven in the same way as in Lemma 4.8.

Lemma 4.10. Let $\mathfrak{X}=\mathfrak{X}_{n}$ be the complexified vector field on $S^{1}$ given by the function $\tilde{h}_{n}(x)=e^{2 \pi i n x}, \tilde{\mathfrak{X}}_{n}=\tilde{h}_{n} \frac{d}{d x}$, n-an integer. Let $\nu^{n} \in B(\mathrm{H}, \Gamma)$ be the Beltrami differential defined in (2.14) for $\mathfrak{x}_{n}$ and let $F_{\nu^{n}}: \mathrm{C} \longrightarrow \mathrm{C}$ be the potential function for $\widehat{\nu^{n}}$ defined in (4.1). Then

$$
F_{\nu^{n}}=\sum_{\gamma \in\left\langle\gamma_{0}\right\rangle \backslash \Gamma}\left(\frac{I_{n} \circ \gamma}{\gamma^{\prime}}-P_{\gamma}^{n}\right)
$$

and the series converges absolutely and locally uniformly on $\mathrm{C}$.

Proof. Let $R(\zeta, z)=\frac{z(z-1)}{\zeta(\zeta-1)(\zeta-z)}$. We have

$$
F_{\nu^{n}}(z)=-\frac{1}{\pi} \iint_{\mathrm{H}} \nu^{n}(\zeta) R(\zeta, z) d \xi d \eta
$$

Since $\nu^{n}(z)=\sum_{\gamma \in\left\langle\gamma_{0}\right\rangle \backslash \Gamma} \nu_{o}^{n}(\gamma(z)) \frac{\overline{\gamma^{\prime}(z)}}{\gamma^{\prime}(z)}$ and all the partial sums of this series are bounded by $\left\|\nu_{o}^{n}\right\|_{\infty}$, we can integrate term by term and obtain

$$
\begin{aligned}
F_{\nu^{n}}(z) & =\sum_{\gamma \in\left\langle\gamma_{0}\right\rangle \backslash \Gamma}-\frac{1}{\pi} \iint_{\mathrm{H}} \nu_{o}^{n}(\gamma(\zeta)) \frac{\overline{\gamma^{\prime}(\zeta)}}{\gamma^{\prime}(\zeta)} R(\zeta, z) d \xi d \eta \\
& =\sum_{\gamma \in\left\langle\gamma_{0}\right\rangle \backslash \Gamma}\left(\frac{I_{n}(\gamma(z))}{\gamma^{\prime}(z)}-P_{\gamma}^{n}(z)\right) .
\end{aligned}
$$

THEOREM 4.11. The Eichler cohomology class $\left[\chi_{\nu^{n}}\right] \in H^{1}\left(\Gamma, \Pi_{2}\right)$ corresponding to the infinitesimal deformation $\varphi_{\left(C_{0}, C_{1}\right)}\left(\mathfrak{X}_{n}\right)$ of the Riemann surface $R=\mathrm{H} / \Gamma$ is given by the cocycle $\chi_{\nu^{n}}$, where

$$
\chi_{\nu^{n}}(\omega)=\frac{F_{\nu^{n}} \circ \omega}{\omega^{\prime}}-F_{\nu^{n}}=\sum_{\gamma \in\left\langle\gamma_{0}\right\rangle \backslash \Gamma}\left(P_{\gamma \circ \omega}^{n}-\frac{P_{\gamma}^{n} \circ \omega}{\omega^{\prime}}\right)
$$

for $\omega \in \Gamma$. (The series converges absolutely and locally uniformly on C.) 
Proof. The function

$$
f_{\gamma}^{n}(z)=\left(\frac{I_{n} \circ \gamma}{\gamma^{\prime}}-P_{\gamma}^{n}\right)(z)=-\frac{1}{\pi} \iint_{\mathrm{H}} \nu_{o}^{n}(\gamma(\zeta)) \frac{\overline{\gamma^{\prime}(\zeta)}}{\gamma^{\prime}(\zeta)} R(\zeta, z) d \xi d \eta
$$

depends only on the right coset of $\gamma$ in $\left\langle\gamma_{0}\right\rangle \backslash \Gamma$. Hence, for $\omega \in \Gamma$,

$$
\begin{aligned}
F_{\nu^{n}} & =\sum_{\gamma \in\left\langle\gamma_{0}\right\rangle \backslash \Gamma} f_{\gamma}^{n}=\sum_{\gamma \in\left\langle\gamma_{0}\right\rangle \backslash \Gamma} f_{\gamma \circ \omega}^{n} \\
& =\sum_{\gamma \in\left\langle\gamma_{0}\right\rangle \backslash \Gamma}\left(\frac{I_{n} \circ(\gamma \circ \omega)}{(\gamma \circ \omega)^{\prime}}-P_{\gamma \circ \omega}^{n}\right) .
\end{aligned}
$$

Since

$$
\frac{F_{\nu^{n}} \circ \omega}{\omega^{\prime}}=\sum_{\gamma \in\left\langle\gamma_{0} \backslash \backslash \Gamma\right.} \frac{f_{\gamma}^{n} \circ \omega}{\omega^{\prime}}=\sum_{\gamma \in\left\langle\gamma_{0} \backslash \backslash \Gamma\right.}\left(\frac{I_{n} \circ(\gamma \circ \omega)}{(\gamma \circ \omega)^{\prime}}-\frac{P_{\gamma}^{n} \circ \omega}{\omega^{\prime}}\right),
$$

we obtain Theorem 4.11.

Remark 4.12. According to [2; Thm 6.10] the quadratic differential $\varphi_{\left(C_{0}, C_{1}\right)}\left(\mathfrak{X}_{n}\right) \in A_{2}\left(\mathrm{H}^{*}, \Gamma\right)$ is equal to $\left(F_{\nu^{\prime \prime}}\right)^{\prime \prime \prime}$. Thus it follows from Lemma 4.10 that $\varphi_{\left(C_{0}, C_{1}\right)}\left(\mathfrak{X}_{n}\right)$ can be obtained by differentiating term by term three times the series $\sum_{\gamma \in\left\langle\gamma_{0}\right\rangle \backslash \Gamma}\left(\frac{I_{n} \circ \gamma}{\gamma^{\prime}}-P_{\gamma}^{n}\right)$. Using Bol's formula (see e.g. [5; p. 515]), we get as a result

$$
\varphi_{\left(C_{0}, C_{1}\right)}\left(\mathfrak{X}_{n}\right)=\sum_{\gamma \in\left\langle\gamma_{0}\right\rangle \backslash \Gamma}\left(I_{n}^{\prime \prime \prime} \circ \gamma\right) \cdot\left(\gamma^{\prime}\right)^{2} .
$$

From the explicit description of $I_{n}$ given in Lemmas 4.4 and 4.5 we get then another proof of Theorem 3.7.

\section{Vector fields on Teichmüller spaces}

Let $R$ be a compact Riemann surface of genus $g \geq 2$ and let $\left(C_{0}, C_{1}\right)$ be a 1pair of geodesics on $R$ (see Definition 2.1).

Let $T(R)$ be the Teichmüller space of $R$. We represent points of $T(R)$ by equivalence classes of quasiconformal maps $f: R \longrightarrow S, S$ being a compact Riemann surface of genus $g$ (see [2; p. 14]). Denote by $[S, f]$ the equivalence class of $f,[S, f] \in T(R)$. 
Given $[S, f] \in T(R)$, consider the simple closed curves $f\left(C_{0}\right)$ and $f\left(C_{1}\right)$ in $S$. Let $C_{0}^{f}, C_{1}^{f}$ be the simple closed geodesics in $S$ in the free homotopy classes of $f\left(C_{0}\right), f\left(C_{1}\right)$ respectively. Then $\left(C_{0}^{f}, C_{1}^{f}\right)$ is a 1-pair of geodesics in $S$. Indeed, since $C_{0}$ and $C_{1}$ have just one intersection point in $R$, so do the curves $f\left(C_{0}\right)$ and $f\left(C_{1}\right)$ in $S$ as well. Hence, by [1; Thm 1.6.7, p. 23], the geodesics $C_{0}^{f}, C_{1}^{f}$ intersect in at most one point. On the other hand the homological intersection number $\left\langle C_{0}, C_{1}\right\rangle$ of $C_{0}$ and $C_{1}$ is \pm 1 . Since $f$ is an orientation preserving homeomorphism, we have

$$
\left\langle C_{0}^{f}, C_{1}^{f}\right\rangle=\left\langle f\left(C_{0}\right), f\left(C_{1}\right)\right\rangle=\left\langle C_{0}, C_{1}\right\rangle= \pm 1 .
$$

Therefore $C_{0}^{f}$ and $C_{1}^{f}$ cannot be disjoint. Thus $C_{0}^{f}$ and $C_{1}^{f}$ intersect in exactly one point and, hence, $\left(C_{0}^{f}, C_{1}^{f}\right)$ is a 1-pair of geodesics. It is also clear that the pair $\left(C_{0}^{f}, C_{1}^{f}\right)$ is independent of the choice of $f$ within the equivalence class $[S, f]$.

Suppose $\mathfrak{X}$ is a smooth vector field on the circle $S^{1}$. Given a point $[S, f] \in T(R)$ let us consider the infinitesimal deformation $\varphi_{\left(C_{0}^{f}, C_{1}^{f}\right)}(\mathfrak{X})$ of the Riemann surface $S$ constructed in Section 2. We look now upon $\varphi_{\left(C_{0}^{f}, C_{1}^{f}\right)}(\mathfrak{X})$ as a tangent vector to the Teichmüller space $T(R)$ at the point $[S, f]$. In that way every smooth vector field $\mathfrak{X}$ on the circle $S^{1}$ induces a vector field $\Phi_{\left(C_{0}, C_{1}\right)}(\mathfrak{X})$ on the Teichmüller space $T(R)$.

In the special case when $\mathfrak{X}=\frac{\widehat{d}}{d x}$ is the constant vector field on $S^{1}, \Phi_{\left(C_{0}, C_{1}\right)}(\mathfrak{X})$ is equal to the Fenchel-Nielsen vector field with respect to the geodesic $C_{0}$ (multiplied by $C_{0}$ :s length $l$ ),

$$
\Phi_{\left(C_{0}, C_{1}\right)}\left(\frac{\widehat{d}}{d x}\right)=l \frac{\partial}{\partial \tau_{C_{0}}},
$$

which has been introduced and studied in [5] and [6]. See also [2; Sec. 8.3].

Geometry of the vector fields $\Phi_{\left(C_{0}, C_{1}\right)}(\mathfrak{X})$ is a subject of a work in progress.

\section{REFERENCES}

1. Buser, P., Geometry and Spectra of Compact Riemann Surfaces, Birkhäuser, Boston, 1992.

2. Imayoshi, Y., Taniguchi, M., An Introduction to Teichmüller Spaces, Springer-Verlag, Tokio, 1992.

3. Kra, I., Automorphic Forms and Kleinian Groups, W. A. Benjamin, Inc., 1972.

4. Milnor, J. W., Remarks on infinite dimensional Lie groups. In: Relativity, Groups and Topology II, Les Houches Session XL, 1983, edited by B. S. de Witt and R. Stora. NorthHolland, Amsterdam, 1984. 
5. Wolpert, S., The Fenchel-Nielsen deformation, Ann. of Math. (2) 115 (1982), 501-528.

6. Wolpert, S., On the symplectic geometry of deformations of a hyperbolic surface, Ann. of Math. (2) 117 (1983), 207-234.

DEPARTMENT OF MATHEMATICS

UPPSALA UNIVERSITY

BOX 480

S-751 06 UPPSALA

SWEDEN.

E-mail: ryszard@math.uu.se 